\title{
INHALT DES VIERTEN HEFTES
}

A u f s ät z e (Articles)

\section{Wolfgang Scheinert}

Joseph Wilhelm Weiler, Julius Weiler und das Anilin: Zur Entwicklungsgeschichte der deutschen Teerfarbenindustrie und der chemischen Technik vor dem Ersten

Weltkrieg. . . . . . . . . . . . . . . . . . . . . . . . 217

Joseph Wilhelm Weiler, Julius Weiler and the Aniline: The History of Development of the German Dye Industry and of the Chemical Technic before the First World War

Konrad Fuchs

Zur Geschichte des Warenhaus-Konzerns I. Schocken Söhne unter besonderer

Berücksichtigung der Jahre seit $1933 \ldots \ldots \ldots \ldots \ldots \ldots . \ldots \ldots \ldots 232$

The History of the Warehouse Combine I. Schocken Söhne with Special Regard to the Years since 1933

B i b liog ra p h i e (Bibliography)

\section{Martina Mundorf}

Bibliographie zur Unternehmensgeschichte und Unternehmerbiographie

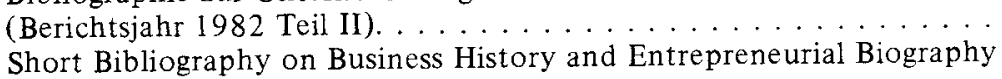

(1982 Year Report Part II)

B u chbesprechungen (Reviews)

Gert Kollmer (Bearb.), Dokumentation zur Organisationsgeschichte der zentralen Arbeitgeberverbände. Hauptstelle Deutscher Arbeitgeberverbände, Verein Deutscher Arbeitgeberverbände, Vereinigung der Deutschen Arbeitgeberverbände (Walther Herrmann). . . . . . . . . . . . . . . . . . . . . . 256

Michaela Blunk, Der Handel des Lübecker Kaufmannes Johan Glandorp an der Wende vom 16. zum 17. Jahrhundert (Harald Witthöft) . . . . . . . . . . 257

Friedrich-Wilhelm Henning, Studien zur Wirtschafts- und Sozialgeschichte Mittelund Ostdeutschlands (Friedrich Richter) . . . . . . . . . . . . 257

Seew Wolffsohn, Wirtschaftliche und soziale Entwicklungen in Brandenburg, Preußen, Schlesien und Oberschlesien in den Jahren 1640-1853 (Konrad Fuchs). . . . . . . 258

Franfois Crouzet, The First Industrialists: The Problem of Origins (David J. Jeremy) . 259 Reinhard Schüren, Staat und ländliche Industrialisierung (Alexander Drexler) . . . . 260 Jochen Putsch, Vom Handwerk zur Fabrik (Jürgen Reulecke) . . . . . . . . . . . 262 Oh! de Cologne, hrsg. v. Herbert Titz (Clemens von Looz-Corswarem). . . . . . . . . . 262 Beate-Carola Padtberg, Rheinischer Liberalismus in Köln während der politischen

Reaktion in Preußen nach $1848 / 49$ (Michael Müller) . . . . . . . . . . . . . . . 264

Mario König/Hannes Siegrist/Rudolf Vetterit, Warten und aufrücken. Die Angestellten in der Schweiz 1870-1950 (Peter Borscheid) . . . . . . . . . . . . 265

Ernst Moritz Spilker, Bayerns Gewerbe 1815-1965 (Wolfgang Zorn) . . . . . . . . 266 Hans Hesselmann, Das Wirtschaftsbürgertum in Bayern 1890-1914 (Wolfgang Zorn) . 268 Wolfgang R. Krabbe, Kommunalpolitik und Industrialisierung (Gerold Ambrosius) . . 269 Gerald D. Feldman u.a. (Hrsg.), Die Erfahrung der Inflation im internationalen

Vergleich (Knut Borchardt). . . . . . . . . . . . . . . . . . 270

Dieter Lindenlaub, Maschinenbauunternehmen in der deutschen Inflation 1919-1923

(Wilhelm Treue) . . . . . . . . . . . . . . . . . . . . . 272

Wolfgang Muth, Berufsausbildung in der Weimarer Republik (Lothar Burchardt) . . . . 273

Hans-Jürgen Perrey, Der Rußlandausschuß der Deutschen Wirtschaft (Konrad Fuchs). 273

Peter Mielmann, Deutsch-chinesische Handelsbeziehungen am Beispiel der Elektroindustrie $1870-1949$ (Horst A. Wessel) . . . . . . . . . . . . . . . . 275

Johannes Barth, Als deutscher Kaufmann in Fernost. Bremen - Tsingtau - Tokyo

1891-1981 (Rolf-Harald Wippich) . . . . . . . . . . . . . . . . . 276

Gabriele Müller-List (Bearb.), Montanmitbestimmung (Hans J. Teuteberg) . . . . . . 277

Max Walter Clauss, Treff punkt Zukunft (Wilhelm Treue) . . . . . . . . . . . . 278 Flachglas AG (Hrsg.), 500 Jahre Flachglas AG: 1487-1987 (Rolf-Jürgen Gleitsmann) 280

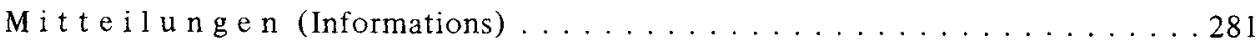


